\title{
Uric acid and coronary artery disease: An elusive link deserving further attention
}

\author{
Simone Biscaglia a ${ }^{\mathrm{a}}$, Claudio Ceconi ${ }^{\mathrm{a}, \mathrm{b}}$, Michele Malagù ${ }^{\mathrm{a}}$, Rita Pavasini ${ }^{\mathrm{a}}$, Roberto Ferrari ${ }^{\mathrm{a}, \mathrm{b}, \mathrm{c}, *}$ \\ a Department of Cardiology, University Hospital of Ferrara, Italy \\ b LTTA Centre, University Hospital of Ferrara, Italy \\ c Maria Cecilia Hospital, GVM Care E' Research, E.S.: Health Science Foundation, Cotignola, Italy
}

\section{A R T I C L E I N F O}

\section{Article history:}

Received 31 July 2015

Accepted 7 August 2015

Available online xxxx

\section{Keywords:}

Uric acid

CAD

Reactive oxygen species

\begin{abstract}
A B S T R A C T
Uric acid is the final product of purine metabolism. Classically it is recognized as the cause of gouty arthritis and kidney stones. Western civilization has increased serum levels of uric acid which is no longer considered a benign plasma solute. It has been postulated and recently demonstrated that it can penetrate cell membrane and exerts damaging intracellular actions such as oxidation and inflammation. These observations have stimulated several epidemiological researches suggesting that hyperuricemia is linked or even provokes hypertension and coronary artery disease. In this review we summarize the current evidences regarding uric acid which contribute in the pathophysiology of coronary artery disease.
\end{abstract}

(c) 2015 Elsevier Ireland Ltd. All rights reserved.

\section{Introduction}

Excess uric acid has been always considered the cause of gouty arthritis and kidney stones, because of accumulation of uric acid crystals due to either overproduction or underexcretion.

Over the last 50 years, however, there has been a reappraisal of the pathological role of excess uric acid in man. The scientific community started to work on the idea that non-crystallized soluble uric acid is responsible for tissue damage, particularly at the level of the liver and the vessels. This was because Talbot et al. observed high incidence of interstitial and vascular disease in autopsied kidney and coined the term "gouty nephropathy" [1]. The same concept was then extended to patients with small deposit of urate crystals or asymptomatic hyperuricemia [2]. Not surprisingly, the majority of nephrologists rejected the idea that soluble uric acid has direct damaging effects to the kidneys not related to kidney stones. There was no clear model or mechanism by which soluble uric acid should damage the vascular tissue or the endothelium of the kidney. At that time, the present well recognized observation that hyperuricemia is associated to hypertension was considered and extended. A new light was then provided to the issue and it was hypothesized that uric acid in excess could cause hypertension and that hypertension itself could be the cause of vascular damage resulting in kidney diseases.

Involvement of hypertension opened several new avenues as any deleterious vascular changes in the heart, brain and kidney could be the consequence of hypertension! Several epidemiologic studies started

\footnotetext{
* Corresponding author at: Azienda Ospedaliero-Universitaria di Ferrara, Via Aldo Moro 8, Cona, 44124 Ferrara, Italy.

E-mail address: fri@unife.it (R. Ferrari).
}

to explore these different hypotheses. Majority of these, but not all, show that uric acid elevation may initiate, or at least be linked, to the endothelial dysfunction associated with stroke, diabetes, cardiovascular disease, kidney disease as well as metabolic syndrome. Going beyond the epidemiologic studies, scientists have also shown that uric acid can penetrate endothelial cells and that it is biologically active, stimulating oxidative stress, inflammation, vasoconstriction, endothelial dysfunction and many other negative actions.

Today the clinical data are on the side that hyperuricemia is associated (if not an independent risk factor) to cardiovascular disease, as well as hypertension, kidney disease and stroke. The final molecular mechanisms for a causal relationship between soluble uric acid and these pathological conditions, however, remain elusive. Animal studies provide suggestion for a mechanistic effect but not a clear picture. As expected, these findings are changing clinicians' and cardiologists' view of asymptomatic hyperuricemia with the temptation to lower serum uric acid levels in order to decrease the risk of morbidity and mortality of cardiovascular disease and new drugs are developed to this end.

In this article we review the evidence in favor or against uric acid to represent a risk factor for cardiovascular disease. Aspects of uric acid metabolism and its involvement in other diseases are addressed in different articles of this supplement.

\subsection{Hyperuricemia in humans: a story from afar}

We, human beings, are all hyperuricemic compared with all other mammals. Only great apes are like us [1]. This is because of a series of genetic mutations which occurred 20 million years ago to the uricase gene, the enzyme that metabolizes uric acid [3]. Mammals with 
functioning uricase have serum urate concentration between 1 and 2 $\mathrm{mg} / \mathrm{dl}$. Our ancestors, before being exposed to modern civilization and western diet, had serum urate level between 2 and $4 \mathrm{mg} / \mathrm{dl}$, a level that today has at least doubled. So there was a clear enzymatic mutation in the human species through the years. The question is why nature provoked such mutation. Nature only favors genetic changes to improve the survival of the species. In the case of uricase, the reason is not at all clear. Three interesting hypothesis have been considered, none mutually exclusive. The first is related to the structural similarity between uric acid and caffeine as well as other neurostimulatory molecules. It is possible that the mutation in uricase increases the level of uric acid which, in turn, allows the alertness and the intelligence needed to achieve our evolutionary advance. The second hypothesis is related to the antioxidant effects of uric acid, mainly to compensate the loss of endogenously produced vitamin $\mathrm{C}$, which occurred just before the loss of uricase enzyme in mammals. The third hypothesis, actually the more relevant for the present discussion, is linked with hypertension. A higher blood pressure was needed by our ancestors to assume an upright position requiring greater cerebral blood perfusion. All the three hypotheses suggest a survival advantage offered by higher level of serum urate, particularly in period when dietary sources of vitamin C and sodium were low. However, as often is the case, in the modern era with abundance of vitamins and salt in our diet, such advantages turn to be a source of disease.

\subsection{Gout and cardiovascular disease: just indulgence to unhealthy lifestyle or...more?}

In general, gouty patients have history of hyperuricemia, the incidence of which is steadily increasing in the western world with wide country variation. Hyperuricemia, in turn, is linked to hypertension, renal disease, metabolic syndrome and coronary or cerebral vascular diseases. Not only, but high levels of uric acid (greater than $7.0 \mathrm{mg} / \mathrm{dl}$ ) also correlate with the usual risk factors such as age, male sex, obesity, dyslipidemia, and insulin resistance. It follows that all these complex relationship between hyperuricemia, risk factors, and cardiovascular diseases are difficult to interpret and the doubt that elevated uric acid is just an epiphenomenon of a rather unhealthy lifestyle does exist. Such a doubt is supported and reinforced by the classical prototype of the gouty patient. Of course, there are always exceptions, but, in general, the typical patient with gout is a middle-aged man, overweight and overindulging in food and alcohol intake! Often this patient presents several associated comorbidities such as hypertension, kidney disease, diabetes mellitus and/or metabolic syndrome. The question, therefore, is whether and when the increased level of soluble uric acid intervenes in such cascade of events. The answer is far from simple and comes from both experimental and clinical studies.

\subsection{Uric acid: just circulating in the blood or penetrating the cells?}

The major skepticism for accepting a causal role of uric acid was related to the uncertainty that an extracellular substance circulating in the blood could exert negative intracellular effects. In 2002, some light into this dilemma was provided by the identification of a specific renal urate-anion exchange transporter, named URAT-1. The transporter, considering the low activity of uricase, is a key factor for the regulation of blood urate levels as it is primarily responsible for renal reabsorption of uric acid in the proximal convoluted tubule. Interestingly, URAT- 1 is also expressed in the vascular endothelial cells as well as in the vascular smooth muscle cells [4]. These observations make an intracellular negative action of uric acid at least possible and provide credibility to the previous studies in rat models showing that hyperuricemia causes microvascular changes independent from hypertension or the presence of urate crystal [5]. Another interesting observation is the link, in rats at least, between inhibition of uricase, rise of serum uric acid and development of hypertension, preventable by lowering uric acid with xanthine oxidase inhibitors or uricosuric agents [6]. Such a link, however, has not been confirmed by other studies [7]. The discrepancy, interestingly, may be related to the experimental model as URAT- 1 is highly expressed in the abdominal aorta but not in the thoracic aorta [8]. As a consequence, responsiveness of vascular tissues to uric acid increase in terms of hypertension development is related to URAT-1 dependent penetration of endothelial and smooth muscle cells.

Another question is whether the intracellular effects of uric acid are also related to development of coronary artery disease (CAD), independently from increased blood pressure. The answer to this question is difficult and far from being completely elucidated as uric acid elevation can exert different and opposite effects. Furthermore, elevated uric acid levels contribute to CAD by interacting with oxygen metabolism, inducing inflammation and endothelial dysfunction.

\subsection{Uric acid and oxidative stress: a double face action, extracellular protection, intracellular damage}

Uric acid exerts opposite effects on oxygen scavenging, according to whether its effects occur extracellularly or intracellularly. Circulating uric acid protects vascular endothelial cells from oxidative stress. It reacts with different oxidants, as singlet oxygen, peroxyl and hydroxyl radicals. The reaction between uric acid and oxidants results in its degradation to specific products such as allantoin, triuret and 6-amminouracil, respectively. It follows that uric acid acts as a vehicle of NO, decreasing vascular tone, increasing blood flow and protecting vascular endothelial cells from external oxidative stress.

This positive effect is true only for the circulating uric acid. When it penetrates the endothelium and the myocyte or when is produced in the cell as terminal step of purine degradation, the effects of uric acid are completely different and from an antioxidant it became a strong pro-oxidant. During the degradation from hypoxanthine to uric acid, xanthine oxidase donates electron to molecular oxygen, through an internal electron transport system, generating reactive oxygen species (ROS), like $\mathrm{O}^{-}$and hydrogen peroxide ( $\mathrm{H} 2 \mathrm{O} 2$ ). $\mathrm{O}^{-}$has a major role in cardiac dysfunction, interacting with several membranes, including the sarcolemma and the mitochondria membranes. In addition, it binds $\mathrm{NO}$ and forms peroxynitrite $\left(\mathrm{OONO}^{-}\right)$, thus reducing $\mathrm{NO}$ bioavailability (Fig. 1).

Even the entry of uric acid into cells causes oxidative stress and reduces NO bioavailability. Oxidative stress is related to activation of nicotinamide adenine dinucleotide phosphate-oxidase (NADPH), while NO bioavailability reduction is related to the block of L-arginine uptake, the NO synthase (NOS) substrate and to the stimulation of L-arginine degradation through arginase. Of course, this results in endothelial dysfunction and vasoconstriction (Fig. 1).

Interestingly, the magnitude of uric acid-related endothelial dysfunction in experimental studies is stronger than its extracellular antioxidant properties [9]. This is because NO inhibits platelets aggregation and adhesion, prevents leukocytes adhesion, reduces intima proliferation, and controls vascular tone. The reduction of its bioavailability induces endothelial dysfunction, through the reduction of NO metabolites, oxidative stress and ultimately an increase of vascular tone. The association between hyperuricemia and endothelial dysfunction has been demonstrated both in rats [10] and in humans, and xanthine oxidase inhibitors treatment has shown to improve endothelial dysfunction [11].

\subsection{Uric acid besides crystal deposits provokes inflammation and activates immunity}

Beside the well-known urate crystals inflammation properties, recent studies show that uric acid stimulates chemokines, such as monocyte chemoattractant protein-1 (MCP-1) and inflammatory markers, such as high-sensitivity $\mathrm{C}$ reactive protein, white blood cells, interleukin-1, 


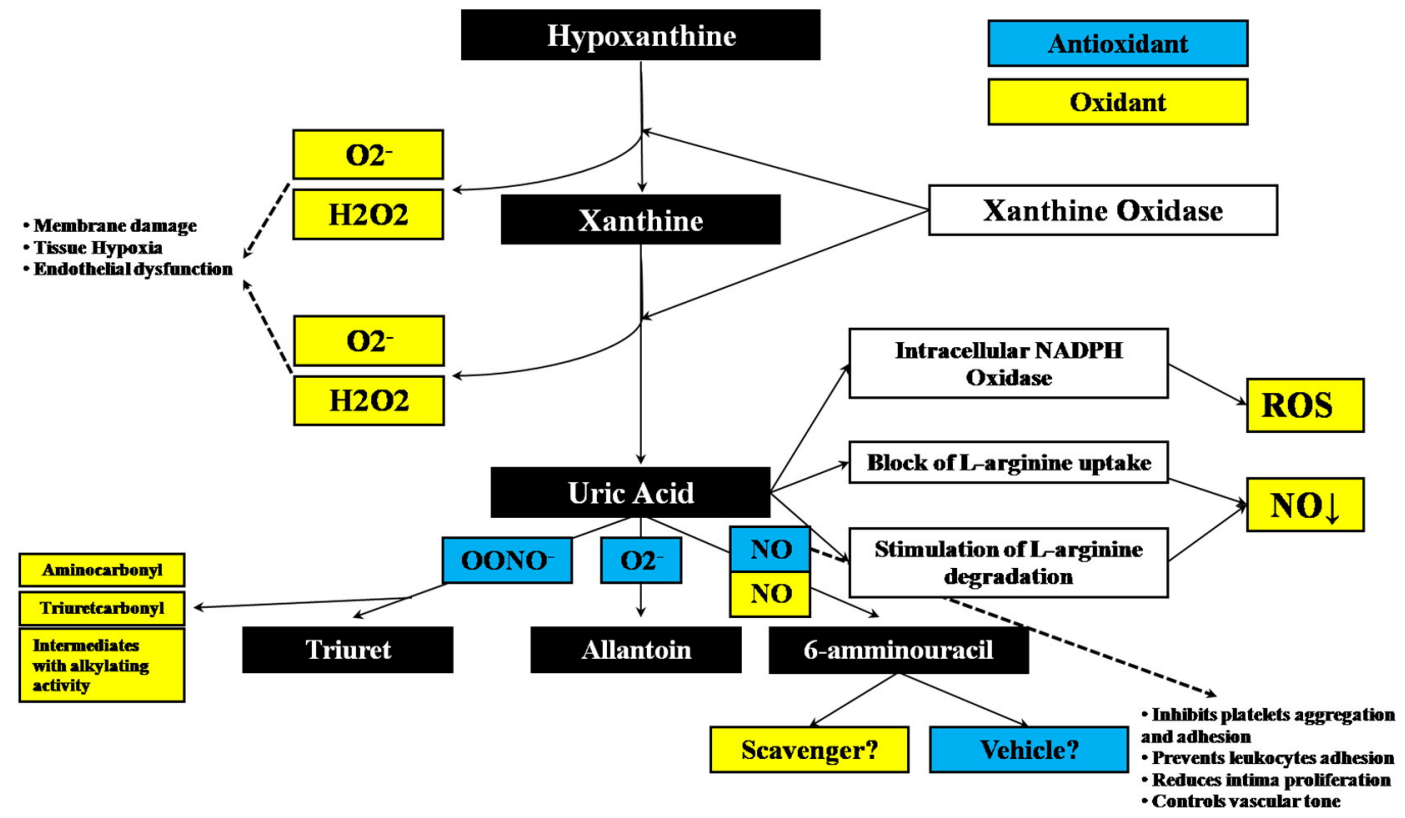

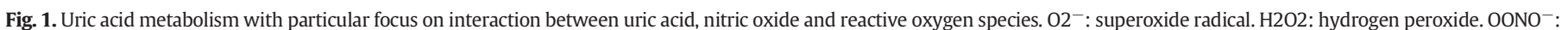
peroxynitrite. NO: nitric oxide. NADPH: nicotinamide adenine dinucleotide phosphate-oxidase. ROS: reactive oxygen species.

interleukin-6, interleukin-10, interleukin-18, endothelin-1 and tumor necrosis factor-alpha [12], all contributing to CAD (Fig. 2).

Intracellular uric acid induces activation of mitogen activated protein kinases (P38) and extracellular signal-regulated kinases (ERK) as well as of nuclear activation factors (NFkB and APO-1), leading to vasoconstriction through mediators and several growth factors (thromboxane, endothelin-1, angiotensin II and platelet-derived growth factor; Fig. 2). In addition, ischemic and dying cells release uric acid, which probably activate the innate immune system through dendritic cells and CD8 + T cells. Interestingly, the induction of heat shock protein70 , one of the local inflammation related proteins, can be blocked by allopurinol [13]. When taken together, these data suggest that uric acid may have a role in the inflammation process occurring during ischemia (Fig. 2).

\subsection{Elevated uric acid and $C A D$ in the clinic: marker or risk factor?}

At present, no definitive conclusion can be drawn as to whether uric acid is an independent predictor of CAD or not. Many studies suggest that uric acid could be a CAD risk factor [14], others reached the opposite conclusion [15]. Even meta-analyses are contradictory [16, 17]. The overall risk of cardiovascular mortality has shown to increase by $12 \%$ for each increase of $1 \mathrm{mg} / \mathrm{dl}$ of uric acid serum levels, and hyperuricemia increases the risk of death in women, as recently confirmed by

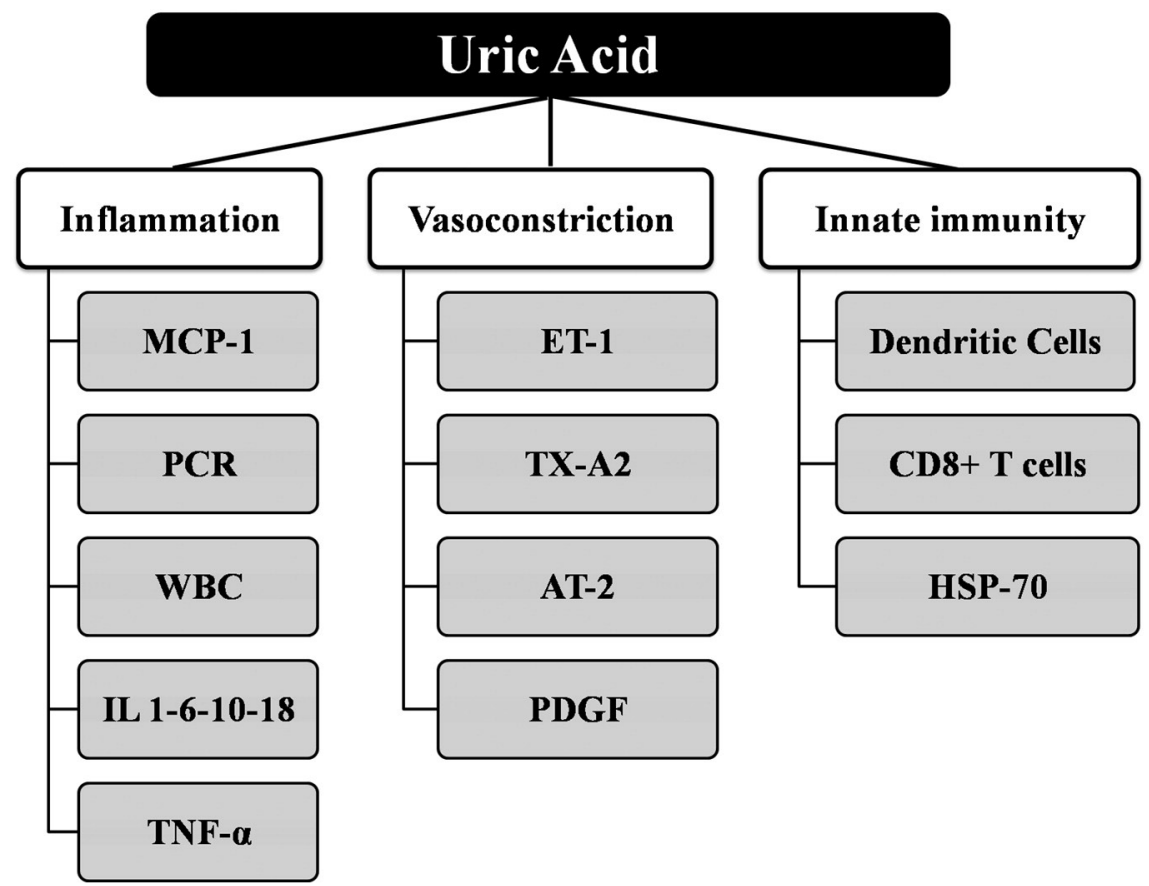

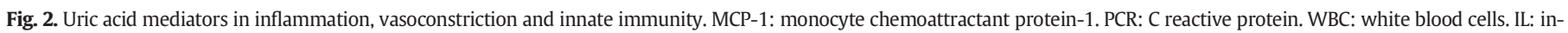

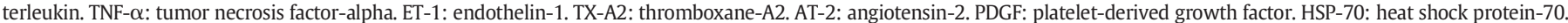


a cross-sectional retrospective study, which included 607 premenopausal women [18]. Other studies evaluated the correlation between coronary artery calcifications (an early marker of CAD) and uric acid levels, but again with opposite results. Neogi et al. reported a clear association [19], while Krishnan et al. could not confirm it [20]. In a retrospective study involving 1901 patients, De Luca et al. showed that uric acid level was not associated both with platelet aggregation and the extent of coronary artery disease [21].

The National Health and Nutrition Survey III (NHANES III) evaluated over 16,000 patients and concluded that uric acid levels in excess of $6 \mathrm{mg} / \mathrm{dl}$ are independent risk factor for CAD [22]. The same conclusion was reached in the systolic elderly program (SHEP), including 4327 patients [23]. So, from multiple studies, it appears plausible that a relationship exists between uric acid and CAD, particularly in patients at high risk for cardiovascular disease and in women. The type of multivariate analysis used to distinguish between dependent and independent risk factors greatly varies between studies adding to the inconsistent conclusions.

To define with certainty a risk factor one has to demonstrate that reducing it, improves prognosis. Prognostic data unfortunately are not available. In a recent randomized crossover trial by Noman et al. [24], 65 patients with stable CAD and provokable ischemia despite optimal anti-ischemic medications, were randomized to receive $600 \mathrm{mg}$ /day of allopurinol or placebo for 6 weeks. Allopurinol administration was associated with a 43-second median increase in exercise time to ST-segment depression ( $\mathrm{p}=0.0002)$, a 58-second median increase in total exercise time $(p=0.0003)$, and a 38-second median increase in time to chest pain $(p=0.001)$. The number of patients is small, but the results are impressing. The authors suggested that the mechanism of the allopurinol anti-ischemic effect may be related not only to uric acid reduction but also to the reduction of oxygen consumption and increase of molecular oxygen in ischemic tissue by a non-specified mechanism. A further examination of the potential role of the anti-ischemic effect of allopurinol was performed in a subsequent study by the same group [25]. In eighty patients with stable CAD and optimal medical therapy, allopurinol $(600 \mathrm{mg} /$ day) or placebo was administered in a crossover design. They observed that allopurinol abolished vascular oxidative stress and improved endothelial-dependent vasodilation. Also the central augmentation index from the pulsed wave analysis was significantly improved with allopurinol. The authors speculated that allopurinol may lead to a possible mortality reduction in patients with CAD. In a subsequent study, Rekhraj et al. randomized 66 patients with ischemic heart disease and left ventricular hypertrophy to $600 \mathrm{mg} /$ day allopurinol versus placebo therapy for 9 months. They found a regression of left ventricular hypertrophy, a reduction in left ventricular endsystolic volume and an improvement in endothelial function and arterial stiffness in allopurinol group [26].

In conclusion, even if current available data are not definitive, several actions of acid uric suggest its involvement in the development and progression of CAD. Perhaps, the most important evidence for a causal relationship between hyperuricemia and vascular disease (hypertension) comes from Feig et al. [27] They demonstrated that lowering uric acid with allopurinol in young hypertensive persons results in reduction in blood pressure.

\section{Conclusion}

The role of uric acid in CAD development remains largely unknown, despite a consistent number of preclinical and clinical studies that tried to elucidate this relationship. Pathophysiological findings suggest a possible role of uric acid in CAD. Clinical results have been more oriented toward hypertension and kidney disease and, in any case, are contradictory. Uric acid could contribute to CAD development through several mechanisms: vasoconstriction, inflammation, oxidative stress and endothelial dysfunction.
Drugs that are currently available to lower serum acid, including allopurinol and others, have not been prognostically investigated in patients with CAD probably because there are not enough proof of concept data to support such studies. At this stage, the hypothesis that hyperuricemia is linked to CAD warrants further investigations involving drugs other than allopurinol which is not free from side effects. It is important to clarify the role of uric acid in CAD, because uric acid levels can be easily modified by drug therapy.

\section{Conflict of interest}

The authors report no relationships that could be construed as a conflict of interest.

\section{Acknowledgment}

This work was supported by a grant from Fondazione Anna Maria Sechi per il Cuore (FASC), Italy. The funders had no role in the study design, data collection and analysis, decision to publish or the preparation of the manuscript.

\section{References}

[1] J.H. Talbott, K.L. Terplan, The kidney in gout, Medicine (Baltimore) 39 (1960) 405-467 (Dec)

[2] J.R. Klinenberg, I. Kippen, R. Bluestone, Hyperuricemic nephropathy: pathologic features and factors influencing urate deposition, Nephron 14 (1) (1975) 88-98.

[3] X.W. Wu, D.M. Muzny, C.C. Lee, C.T. Caskey, Two independent mutational events in the loss of urate oxidase during hominoid evolution, J. Mol. Evol. 34 (1) (1992) 78-84 (Jan).

[4] A. Enomoto, H. Kimura, A. Chairoungdua, Y. Shigeta, P. Jutabha, S.H. Cha, M. Hosoyamada, M. Takeda, T. Sekine, T. Igarashi, H. Matsuo, Y. Kikuchi, T. Oda, K. Ichida, T. Hosoya, K. Shimokata, T. Niwa, Y. Kanai, H. Endou, Molecular identification of a renal urate anion exchanger that regulates blood urate levels, Nature 417 (6887) (2002) 447-452 (May 23).

[5] N.L. Edwards, The role of hyperuricemia in vascular disorders, Curr. Opin. Rheumatol. 21 (2) (2009) 132-137 (Mar).

[6] M. Mazzali, J. Kanellis, L. Han, L. Feng, Y.Y. Xia, Q. Chen, D.H. Kang, K.L. Gordon, S. Watanabe, T. Nakagawa, H.Y. Lan, R.J. Johnson, Hyperuricemia induces a primary renal arteriolopathy in rats by a blood pressure-independent mechanism, Am. J. Physiol. Renal Physiol. 282 (6) (2002) F991-F997 (Jun).

[7] V. Kurra, A. Eräranta, P. Jolma, T.I. Vehmas, A. Riutta, E. Moilanen, A. Tahvanainen, J. Kalliovalkama, O. Niemelä, J. Myllymäki, J. Mustonen, I. Pörsti, Hyperuricemia, oxidative stress, and carotid artery tone in experimental renal insufficiency, Am. J. Hypertens. 22 (9) (2009) 964-970, http://dx.doi.org/10.1038/ajh.2009.109 (Sep, Epub 2009 Jun 11).

[8] M. Kanbay, M. Segal, B. Afsar, D.H. Kang, B. Rodriguez-Iturbe, R.J. Johnson, The role of uric acid in the pathogenesis of human cardiovascular disease, Heart 99 (11) (2013) 759-766 (Jun).

[9] C. Gersch, S.P. Palii, K.M. Kim, A. Angerhofer, R.J. Johnson, G.N. Henderson, Inactivation of nitric oxide by uric acid, Nucleosides Nucleotides Nucleic Acids 27 (8) (2008) 967-978 (Aug)

[10] U.M. Khosla, S. Zharikov, J.L. Finch, T. Nakagawa, C. Roncal, W. Mu, K. Krotova, E.R. Block, S. Prabhakar, R.J. Johnson, Hyperuricemia induces endothelial dysfunction, Kidney Int. 67 (5) (2005) 1739-1742 (May).

[11] J. George, E. Carr, J. Davies, J.J. Belch, A. Struthers, High-dose allopurinol improves endothelial function by profoundly reducing vascular oxidative stress and not by lowering uric acid, Circulation 114 (23) (2006) 2508-2516 (Dec 5).

[12] C. Ruggiero, A. Cherubini, A. Ble, A.J. Bos, M. Maggio, V.D. Dixit, F. Lauretani, S. Bandinelli, U. Senin, L. Ferrucci, Uric acid and inflammatory markers, Eur. Heart J. 27 (10) (2006) 1174-1181 (May).

[13] Y. Shi, J.E. Evans, K.L. Rock, Molecular identification of a danger signal that alerts the immune system to dying cells, Nature 425 (6957) (2003) 516-521 (Oct 2).

[14] M. Duran, N. Kalay, M. Akpek, O. Orscelik, D. Elcik, A. Ocak, M.T. Inanc, H.A. Kasapkara, A. Oguzhan, N.K. Eryol, A. Ergin, Kaya MG High levels of serum uric acid predict severity of coronary artery disease in patients with acute coronary syndrome, Angiology 63 (6) (2012) 448-452 (Aug).

[15] V.C. Kok, J.T. Horng, W.S. Chang, Y.F. Hong, T.H. Chang, Allopurinol therapy in gout patients does not associate with beneficial cardiovascular outcomes: a populationbased matched-cohort study, PLoS ONE 9 (6) (2014) (Jun 4, e99102).

[16] J.G. Wheeler, K.D. Juzwishin, G. Eiriksdottir, V. Gudnason, J. Danesh, Serum uric acid and coronary heart disease in 9458 incident cases and 155,084 controls: prospective study and meta-analysis, PLoS Med. 2 (3) (2005) (Mar, e76).

[17] S.Y. Kim, J.P. Guevara, K.M. Kim, H.K. Choi, D.F. Heitjan, D.A. Albert, Hyperuricemia and coronary heart disease: a systematic review and meta-analysis, Arthritis Care Res. 62 (2) (2010) 170-180 (Feb)

[18] J.W. Zhang, L.J. He, S.J. Cao, Q. Yang, S.W. Yang, Y.J. Zhou, Association of serum uric acid and coronary artery disease in premenopausal women, PLoS ONE 9 (9) (2014) (Sep 3, e106130). 
[19] T. Neogi, R. Terkeltaub, R.C. Ellison, S. Hunt, Y. Zhang, Serum urate is not associated with coronary artery calcification: the NHLBI Family Heart Study, J. Rheumatol. 38 (1) (2011) 111-117 (Jan).

[20] E. Krishnan, B.J. Pandya, L. Chung, O. Dabbous, Hyperuricemia and the risk for subclinical coronary atherosclerosis-data from a prospective observational cohort study, Arthritis Res. Ther. 13 (2) (2011) R66 (Apr 18).

[21] G. De Luca, G.G. Secco, M. Santagostino, L. Venegoni, S. Iorio, E. Cassetti, M. Verdoia, L. Coppo, C. Di Mario, G. Bellomo, P. Marino, Novara Atherosclerosis Study Group (NAS), Uric acid does not affect the prevalence and extent of coronary artery disease. Results from a prospective study, Nutr. Metab. Cardiovasc. Dis. 22 (5) (2012) 426-433 (May).

[22] H.J. Ward, Uric acid as an independent risk factor in the treatment of hypertension, Lancet 352 (9129) (1998) 670-671 (Aug 29).

[23] L.V. Franse, M. Pahor, M. Di Bari, R.I. Shorr, J.Y. Wan, G.W. Somes, W.B. Applegate, Serum uric acid, diuretic treatment and risk of cardiovascular events in the Systolic
Hypertension in the Elderly Program (SHEP), J. Hypertens. 18 (8) (2000) 1149-1154 (Aug).

[24] A. Noman, D.S. Ang, S. Ogston, C.C. Lang, A.D. Struthers, Effect of high-dose allopurinol on exercise in patients with chronic stable angina: a randomised, placebo controlled crossover trial, Lancet 375 (9732) (2010) 2161-2167 (Jun 19).

[25] N.S. Rajendra, S. Ireland, J. George, J.J. Belch, C.C. Lang, A.D. Struthers, Mechanistic insights into the therapeutic use of high-dose allopurinol in angina pectoris, J. Am. Coll. Cardiol. 58 (8) (2011) 820-828 (Aug 16).

[26] S. Rekhraj, S.J. Gandy, B.R. Szwejkowski, M.A. Nadir, A. Noman, J.G. Houston, C.C. Lang, J. George, A.D. Struthers, High-dose allopurinol reduces left ventricular mass in patients with ischemic heart disease, J. Am. Coll. Cardiol. 61 (9) (2013) 926-932 (Mar 5).

[27] D.I. Feig, R.J. Johnson, Hyperuricemia in childhood primary hypertension, Hypertension 42 (3) (2003) 247-252 (Sep). 